Pesq. Vet. Bras. 36(11):1121-1126, novembro 2016 DOI: $10.1590 / \mathrm{S} 0100-736 \mathrm{X} 2016001100011$

\title{
Análise do extrato aquoso de Arachis hipoagea L. no combate à dislipidemia e ao ganho ponderal de ratos Wistar submetidos à dieta hiperlipídica ${ }^{1}$
}

\begin{abstract}
Thárcia K.B. de Oliveira ${ }^{2,3 *}$, Francisco de A.C. Almeida², Maria Priscila M.M. Falcão ${ }^{3}$, Ana Janaina J.M. de Lemos-Jordão ${ }^{3,4}$, Khatarina R. de L.P. Ramos ${ }^{5}$ e Juliana F. da Silva ${ }^{2}$

ABSTRACT.- Oliveira T.K.B., Almeida F.A.C., Falcão M.P.M.M., Lemos-Jordão A.J.J.M., Ramos K.R.L.P.\& Silva J.F. 2016. [Analysis of the aqueous extract of Arachis hipoagea L. to reduce dyslipidemia and weight gain in Wistar rats with high fat diet.] Análise do extrato aquoso de Arachis hipoagea L. no combate à dislipidemia e ao ganho ponderal de ratos Wistar submetidos à dieta hiperlipídica. Pesquisa Veterinária Brasileira 36(11):1121-1126. Programa de Pós-Graduação em Engenharia Agrícola, Universidade Federal de Campina Grande, Av. Aprígio Veloso 882, Campina Grande, PB, Brazil. E-mail: tharcia_kiara@hotmail.com

The aim of this study was to evaluate the effect of Aqueous Extract from Peanut (EAA) in weight, serum biochemistry and liver histology of Wistar rats with normal hearing and a high fat diet. The survey was conducted using 40 male Wistar rats divided into four groups (n=0): GA (high fat diet), GB (fat diet+EAA), GC (normolipídica diet), and GD (normolipídica diet+EAA). After 8 weeks, the rats were euthanized and blood samples were collected to evaluate biochemical data (total cholesterol and its fractions, triglycerides, urea, creatinine, AST, ALT and glucose) and liver fragments for histological analysis. The animals of the GB group had a lower weight gain when compared with GA (XGB versus $X G A=p<0.05$ ), but CG and GD did not obtain statistical differences. The rats that received EAA had a reduction in cholesterol levels (XGB $=$ versus $X G A=p<0.05$ vs. $X G A$ and $X G D=p<0.01$ ), triglycerides $(=X G B$ versus $X G A$ and $=X G D$ versus $X G A=p<0,001)$ and more discreetly ALT levels. Blood glucose, urea and creatine remained within the reference values. Liver samples analyzed, the rats of different groups showed no histopathological changes. In conclusion, the EAA had preventive effects on weight gain and dyslipidemia.
\end{abstract}

INDEX TERMS: Arachis hypogaea L., dyslipidemia, rat.

RESUMO.- 0 objetivo desse estudo foi avaliar os efeitos do Extrato Aquoso de Amendoim (EAA) no peso, bioquímica sérica e na histologia hepática de ratos Wistar submetidos a dietas normo e hiperlipídicas. A pesquisa foi realizada uti-

\footnotetext{
${ }^{1}$ Recebido em 8 de dezembro de 2015.

Aceito para publicação em 27 de maio de 2016

${ }^{2}$ Programa de Pós-Graduação em Engenharia Agrícola, Universidade Federal de Campina Grande (UFCG), Av. Aprígio Veloso 882, Bodocongó Campus I, Bloco CM, 1ำ Andar Campina Grande, PB 58429-140, Brasil. *Autora para correspondência: tharcia_kiara@hotmail.com

${ }^{3}$ Faculdade de Ciências Médicas de Campina Grande (FCM), Av. Senador Argemiro de Figueiredo 1901, Itararé, Campina Grande, PB 58411-020, Brasil.

${ }^{4}$ Unidade Acadêmica de Ciências Médicas, UFCG, R. Duque de Caxias, Campina Grande, PB 58400-506, Brasil.

${ }^{5}$ Centro de Ciências Biológicas e da Saúde, Universidade Estadual da Paraíba (UEPB), Rua Baraúnas 351, Universitário, Campina Grande, PB 58429-500, Brasil.
}

lizando 40 ratos Wistar machos, divididos em quatro grupos ( $\mathrm{n}=10)$ : GA (dieta hiperlipídica), GB (dieta hiperlipídica +EAA), GC (dieta normolipídica) e GD (dieta normolipídica +EAA). Após 8 semanas, os animais foram eutanasiados e foram coletadas amostras sanguíneas para a avaliação de dados bioquímicos (Colesterol total e suas frações, triglicerídeos, uréia, creatinina, AST, ALT e glicemia) e fragmentos do fígado para análise histológica. Os animais do grupo GB tiveram um ganho de peso inferior quando comparados ao $\mathrm{GA}(\mathrm{XGB}=$ versus $\mathrm{XGA}=\mathrm{p}<0,05$ ), já os grupos $\mathrm{GC}$ e GD não obtiveram diferenças estatísticas. Os animais que receberam o EAA tiveram uma redução nos níveis de colesterol $(\mathrm{XGB}=$ versus $\mathrm{XGA}=\mathrm{p}<0,05$ e $\mathrm{XGD}=$ versus $\mathrm{XGA}=\mathrm{p}<0,01)$, dos triglicerídeos $(\mathrm{XGB}=$ versus $\mathrm{XGA}$ e $\mathrm{XGD}=$ versus $\mathrm{XGA}=$ $\mathrm{p}<0,001)$ e mais discretamente dos níveis de ALT. A glicemia, uréia e creatina permaneceram dentro dos valores de referência. As amostras hepáticas analisadas, dos ratos dos 
diferentes grupos, não apresentaram alterações histopatológicas. Conclui-se que O EAA apresentou efeitos preventivos sobre o ganho ponderal e dislipidemia.

TERMOS DE INDEXAÇÃO: Arachis hypogaea L., dislipidemia, ratos.

\section{INTRODUÇÃO}

No Brasil, estima-se que cerca de 10 milhões de pessoas apresentam problemas de saúde devido aos maus hábitos alimentares. Estudos demonstram a relação estreita entre a dieta e o estado de saúde do indivíduo, o que tem gerado um aumento no interesse por uma alimentação saudável, que conduza a redução da gordura corporal e melhora do perfil lipídico, diminuindo consequentemente o índice de doenças ateroscleróticas (Alper 2002, Machado et al. 2003).

Arachis hypogaea L. (amendoim) é considerada um alimento altamente energético, composto por 48,7\% de óleos, sendo estes representados por $80 \%$ de ácidos graxos insaturados - oléico $(41,90 \%)$, linoléico $(37,51 \%)$, palmítico $(13,30 \%)$, esteárico $(3,67 \%)$ e araquidônico (1,85\%). Além disso, é uma ótima fonte de proteína vegetal, fibra dietética, antioxidantes, minerais (selênio, magnésio e manganês) e fitoquímicos, como o resveratrol e outros polifenóis (Paschoal et al. 2007, Basode et al. 2012, Sebei et al. 2013). Os antioxidantes têm como propriedade reduzir o estresse oxidativo e consequentemente, o índice de eventos ateroscleróticos (Naz et al. 2014).

Mediante tal composição de $A$. hypogaea, pesquisadores do LAPPA (Laboratório de Armazenamento e Processamento de Produtos Agrícolas) da Universidade Federal de Campina Grande/PB desenvolveram o extrato aquoso de amendoim (EAA) - "leite de amendoim" -, um alimento palatável de baixo custo e de fácil acesso, com potencial de prevenir e combater doenças, colaborando para o alcance de uma vida saudável (Albuquerque et al. 2014, Almeida et al. 2014).

Existem algumas referências a respeito das características de A. hipogaea, entretanto não foram identificados os aspectos histofisiológicos relacionados aos efeitos na prevenção do sobrepeso e da dislipidemia, dados esses importantes, tendo em vista os conhecidos efeitos adversos da terapia medicamentosa empregada nesses casos atualmente (Basode et al 2012, Albuquerque et al. 2014). Baseado neste contexto, o estudo teve como objetivo avaliar os efeitos do extrato aquoso de amendoim no peso, bioquímica sanguínea e na histologia hepática de ratos Wistar submetidos a dietas normo e hiperlipídicas.

\section{MATERIAL E MÉTODOS}

Material de estudo. Para a elaboração do extrato aquoso de amendoim (leite de amendoim) foi utilizada uma máquina desenvolvida no LAPPA, denominada DiaMilk. Todo o processo foi realizado seguindo a sequência em que após a retirada da "pele" do grão, os mesmos foram lavados em água corrente e em seguida, triturados na DiaMilk - em água aquecida a 600C. Posteriormente, o extrato sofreu branqueamento a temperatura de $98^{\circ} \mathrm{C}$ por um tempo de 10 minutos finalizando o processo (Almeida et al. 2014).

O leite foi armazenado em potes de $100 \mathrm{ml}$ e acondicionado em freezer à temperatura de $-18^{\circ} \mathrm{C}$. Antecedendo a administração por gavagem do extrato aquoso de amendoim aos animais, o mesmo foi descongelado por cerca de 1 hora em temperatura ambiente.
Animais experimentais. Foram utilizados 40 ratos Wistar machos, provenientes da colônia de criação do Biotério da Faculdade de Ciências Médicas de Campina Grande (FCM/CG). Os animais foram alojados em gaiolas de polipropileno com dimensão de 430x430x200mm (CxLxA) em um ambiente com temperatura de $23 \pm 1^{\circ} \mathrm{C}$ e ciclo de luz/escuridão (12-12h) recebendo água e dieta ad libitum. 0 experimento foi iniciado quando os animais completaram em média 240 dias de vida, sendo considerados adultos, dos quais foram selecionados ratos com peso entre $250 \mathrm{e}$ $300 \mathrm{~g}$ em todos os grupos experimentais.

Os animais foram divididos em quatro grupos $(n=10)$, identificados conforme a dieta oferecida: GA referente ao grupo controle com dieta de ração hiperlipídica; GB correspondente ao grupo experimental com dieta de ração hiperlipídica associado ao de EAA por via oral ( $2 \mathrm{ml} / \mathrm{dia})$; GC compondo o grupo experimental com dieta de ração comercial; GD referente a administração de ração comercial associada ao EAA (2ml/dia). Todos os grupos receberam água ad libitum.

Desenho do estudo. A dieta hiperlipídica foi obtida da seguinte forma: A cada $90 \mathrm{~g}$ da ração padrão sob a forma de pequenos cubos, foi acrescentado $10 \mathrm{~g}$ do óleo de soja. Os cubos permaneceram em contato com o óleo até este ser absorvido completamente somente então foram oferecidos aos animais dos grupos A e B (Araújo 2007).

A duração do experimento foi de 08 semanas e a administração do extrato foi feita diariamente de forma única, apenas ao final do dia. Nesse período realizou-se o acompanhamento do comportamento biológico dos animais quanto a alterações no peso, isolamento, ou diarréia, além da aferição diária do peso dos animais.

Ao final do experimento os ratos de todos os grupos foram submetidos à anestesia com solução aquosa a $2 \%$ de cloridrato de 2-(2,6 xilidino)-5, 6-(dihidro)-4 - H-1,3 tiazina (Rompun ${ }^{\circledR}$ ) diluído em 1:1 em Ketamina (Fancotar ${ }^{\circledR}$ ), via intraperitoneal na dosagem de $1,0 \mathrm{ml} / \mathrm{kg}$ de peso e em seguida coletou-se $5 \mathrm{ml}$ de sangue de cada animal para a análise bioquímica.

Análises bioquímicas. Cada amostra sanguínea foi centrifugada e o plasma utilizado para os exames laboratoriais. Para o estudo da função renal foi realizada a dosagem sérica de uréia e creatina, para a avaliação hepática (diagnóstico de lesão no fígado) foram dosadas AST (aspartato aminotrasferase) e ALT (alanina aminotrasferase) e a fim determinar o perfil lipídico, identificou-se LDL (Low-densitylipoprotein), HDL (High-densitylipoprotein), colesterol total e os triglicerídeos, ainda foi analisada a glicemia. Os parâmetros bioquímicos dos animais submetidos às diferentes dietas foram comparados seguindo a análise de Lapchik et al. (2010) e Melo et al. (2012). Em seguida, procedeu-se a abertura total do abdome para análise macroscópica e retirada de fragmentos do fígado. 0 maior lobo hepático (lobo esquerdo) foi seccionado em seu maior diâmetro; o fragmento obtido foi fixado em formaldeído a $10 \%$ tamponado, permanecendo no mesmo por 24 horas. Posteriormente, o órgão foi processado para inclusão em parafina e os cortes submetidos à técnica de coloração pela Hematoxilina - Eosina (HE) para análise de rotina histopatológica.

Análises histopatológicas e histoquímica. Para quantificação de lipídeos por histoquímica, foi utilizado o corante Oil-Red-0, fragmentos retirados da mesma região anatômica hepática de todas as ratas de cada grupo foram fixados utilizando-se formaldeído a 4\% com tampão fosfato 0,1M por duas horas e logo após as amostras foram tratadas com solução de sacarose nas concentrações de 15 e $30 \%$ por 24 horas cada, respectivamente, sob resfriamento com N-hexano antes de ser congelada em nitrogênio líquido e acondicionado em freezer $-80^{\circ} \mathrm{C}$. Em seguida o material foi cortado em criostato RM 2035 (Reichert S, Leica), e submetido ao procedimento para coloração específica Oil-Red-O durante 15 minutos. 
Além do estudo histológico, foi realizada a análise qualitativa de gordura hepática dos animais dos Grupos GA, GB, GC e GD com a identificação de elementos que possam avaliar o potencial antiaterogênico do extrato aquoso de amendoim.

Análises estatísticas. Para pesos e parâmetros bioquímicos os resultados foram analisados através do Programa Computacional Assistat, versão 7.4 beta, subtendo os dados a Análise de Variância $(\mathrm{P}<0,05)$ considerando o delineamento inteiramente casualizado (DIC). Cada grupo foi repetido 10 vezes e as médias, quando necessário, foram comparadas pelo o teste de Tukey $(\mathrm{P}<0,05)$.

Os dados referentes ao Oil-Red-O foram submetidos à análise de variância (ANOVA) e testes post-hoc de Dunnett ou Tukey. Sendo considerados significantes os valores com probabilidade menor que 0,05 , para construção de gráficos pelo software GraphPad Prism, version 5.

Ética no uso de animais. Todos os procedimentos com os animais obedeceram às normas vigentes do Conselho Nacional de Experimentação Animal CONCEA e pelo Colégio Brasileiro de Experimentação Animal - COBEA, seguindo a Diretriz Brasileira de Prática para o Cuidado e a Utilização de Animais para Fins Científicos e Didáticos - DBPA. O experimento só foi realizado após aprovação da Comissão de Ética no Uso de Animais - CEUA do Centro de Ensino Superior e Desenvolvimento - CESED.

\section{RESULTADOS}

Durante o experimento com EAA não foram observados sinais clínicos de toxicidade e nenhuma morte foi registrada. As medidas referentes aos perfis bioquímicos relacionados aos animais que receberam dieta hiperlipídica estão apresentadas no Quadro 1. Os animais do grupo GB apresentaram diminuição significativa do colesterol, triglicerídeos e VLDL quando comparados ao grupo controle (GA), sendo que os dois últimos parâmetros tiveram uma diferença mais expressiva $(p<0,01)$. HDL não apresentou diferença significativa quando comparados. Os valores de LDL foram calculados de acordo com a equação de Friedewald, que apesar do aumento de 19,4mg/dl no LDL do GA, não foi observado diferença estatística, entre os grupos GA e GB.

Os resultados das dosagens da uréia e AST não apresentaram diferença significativa entre os grupos, entretanto os valores médios de AST ficaram acima dos valores de referência (39-92). Os valores do ALT foram distintos estatisticamente a $1 \%$ de probabilidade; enquanto os de creatinina

\section{Quadro 1. Análises bioquímicas dos ratos que receberam dieta hiperlipídica, com e sem adição de $2 \mathrm{ml}$ de EAA e do grupo controle}

\begin{tabular}{lcccc}
\hline \multicolumn{1}{c}{ Parâmetros } & \multicolumn{4}{c}{ Grupos } \\
\cline { 2 - 5 } & $\mathrm{GA}_{\text {hiperlipídica }}$ & $\mathrm{GB}_{\text {hiperlipídica+extrato }}$ & 1 & $\mathrm{p}$ \\
\hline Colesterol total (mg/dL) & $66,8 \pm 10,9$ & $52,4 \pm 8,3$ & $46-92$ & $0,0209^{*}$ \\
HDL (mg/dL) & $37,4 \pm 7,24$ & $34,9 \pm 3,9$ & - & $\mathrm{ns}$ \\
LDL (mg/dL) & $34,0 \pm 12,35$ & $14,6 \pm 4,84$ & - & $\mathrm{ns}$ \\
Triglicerídeos (mg/dL) & $130,0 \pm 11,94$ & $80,25 \pm 13,34$ & $77-110$ & $<0,01^{* *}$ \\
VLDL (mg/dL) & $26,00 \pm 2,1$ & $16,05 \pm 2,35$ & $15-22$ & $<0,01^{* *}$ \\
AST (U/L) & $89 \pm 12,0$ & $95 \pm 20,2$ & $39-92$ & $\mathrm{~ns}$ \\
ALT (U/L) & $53,75 \pm 10,8$ & $41,87 \pm 8,5$ & $17-50$ & $0,029^{*}$ \\
Uréia(mg/dL) & $38,4 \pm 1,9$ & $34,8 \pm 2,5$ & $32-54$ & $0,002^{* *}$ \\
Creatinina (mg/dL) & $0,63 \pm 0,04$ & $0,62 \pm 0,09$ & & $\mathrm{~ns}$ \\
Glicemia(mg/dL) & $126,8 \pm 12,5$ & $116,0 \pm 18,0$ & $85-132$ & $\mathrm{~ns}$
\end{tabular}

** Significativo a $1 \%$ de probabilidade $(\mathrm{p}<0,01)$; * significativo a $5 \%$ de probabilidade $(\mathrm{p}<0,05) ; \mathrm{ns}=$ não significativo. Valor de Referência ${ }^{1}$.
Quadro 2. Peso inicial e final dos animais que receberam dietas hiperlipídica e ração padrão durante o experimento

\begin{tabular}{lcc}
\hline \multicolumn{1}{c}{ Grupo } & \multicolumn{2}{c}{ Peso } \\
\cline { 2 - 3 } & Inicial & Final \\
\hline GA $_{\text {hiperlipídica }}$ & $383^{\mathrm{a}}$ & $424^{\mathrm{a}}$ \\
$\mathrm{GB}_{\text {hiperlipídica+extrato }}$ & $389^{\mathrm{a}}$ & $385^{\mathrm{b}}$ \\
$\mathrm{CV \%}$ & 7,77 & 8,05 \\
$\mathrm{P}$ & $\mathrm{Ns}$ & $0.0143^{*}$ \\
GC padrão & $392,0^{\mathrm{a}}$ & $416,5^{\mathrm{a}}$ \\
GD padrão +extrato & $394,9^{\mathrm{a}}$ & $382,4^{\mathrm{a}}$ \\
CV\% & 9,78 & 9,51 \\
P & $\mathrm{Ns}$ & $\mathrm{Ns}$
\end{tabular}

*Significativo a 5\% de probabilidade $(\mathrm{p}<0,05)$; ns = não significativo. As médias seguidas pela mesma letra na coluna (referentes GA com GB e GC com GD), não diferem estatisticamente entre si.

e glicose a $5 \%(\mathrm{p}<0,05)$, mas por outro lado estes valores encontram-se entre os de referências (85-132).

As médias dos pesos iniciais dos animais dos quatro grupos não apresentaram diferença estatística quando comparadas entre si (Quadro 2). Entretanto, a média do peso corporal final dos animais do grupo que recebeu dieta hiperlipídica, mostraram diferenças estatísticas (Peso inicial $=383 \mathrm{~g}$ versus peso final $=424 \mathrm{~g} \mathrm{p}<0,05$ ).

Apesar da diferença estatística de peso final para os grupos GA e GB (Fig.1A,B), o consumo de ração hiperlipídica dos grupos foram semelhantes, exceto nas semanas 3 e 4 onde se observou um consumo maior do grupo GB, conforme dados da Figura 2A,B. Apesar dessa variação do padrão alimentar, não houve diferença significativa entre as médias gerais quanto à ingestão de ração entre os grupos GA e GB (ns - CV\% =10,06).

As análises de peso inicial e final dos grupos de animais GC e GD não apresentaram diferenças estatísticas significativas (Quadro 2).

Em análise aos valores absolutos de peso dos animais tomados a cada 15 dias observam-se mediante os dados da Figura 1A,B para os primeiros 45 dias, comportamento si-

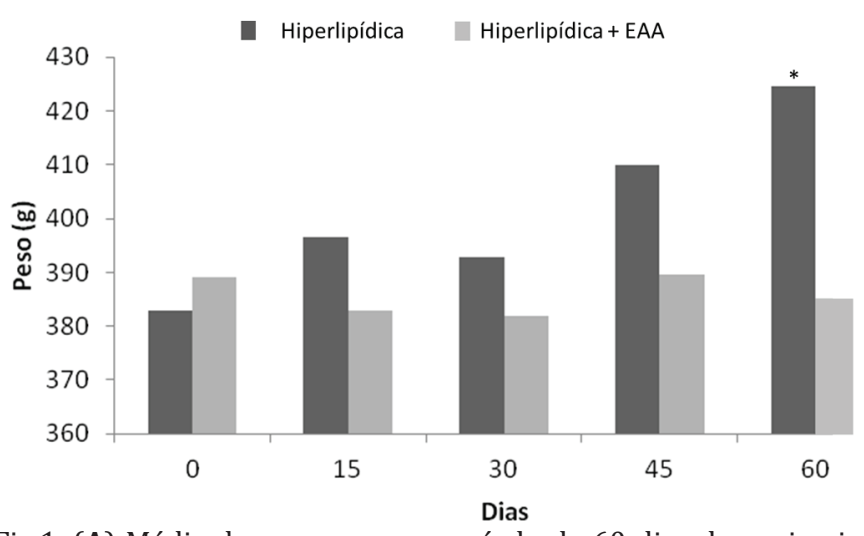

Fig.1. (A) Média de peso por um período de 60 dias dos animais que receberam: (GA) dietahiperlipídica e dieta hiperlipídica suplementada com EAA. *Diferença estatística significativa a $5 \%$ de probabilidade $(\mathrm{p}<0,05)$ entre os grupos GA e GB aos 60 dias. (B) Média de peso por um período de 60 dias dos animais que receberam (GB) normolipídica e dieta normolipídica suplementado com EAA. *Diferença estatística significativa a 5\% de probabilidade $(p<0,05)$ entre os grupos GA e GB aos 60 dias. 


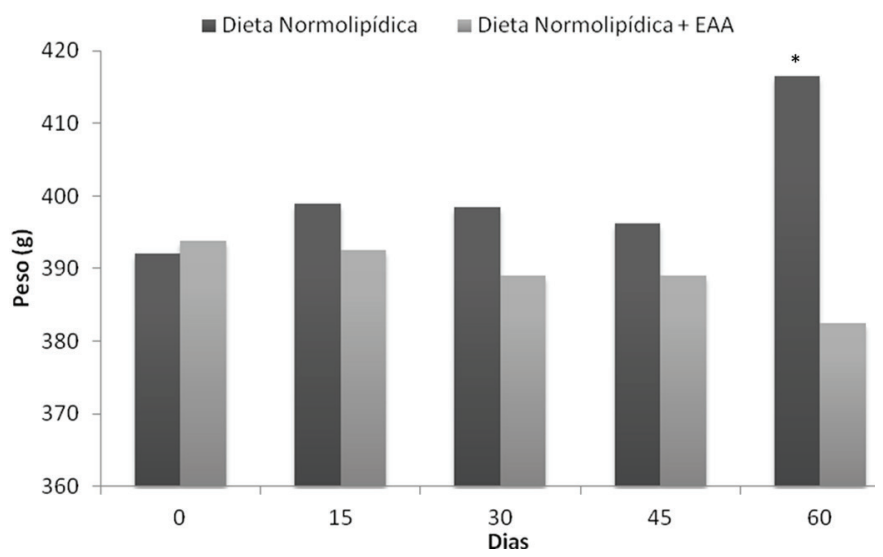

Fig.2 (A) Consumo médio semanal da dieta oferecida aos grupos: Dieta Hiperlipídica (GA) e Hiperlipídica + EAA (GB). (B) Consumo médio semanal da dieta oferecida aos grupos: Dieta Normolipídica (GC) e Dieta Normolipídica + EAA (GD).

milar de ganho de peso dos animais alimentados com a ração padrão (GC) e os que receberam esta ração suplementada com o extrato aquoso de amendoim (GD), mas com maior ganho de peso para os animais alimentados somente com a ração padrão. Nos quinze dias subsequentes, a diferença foi mais expressiva entre estes grupos, no entanto sem que a estatística detectasse.

Os resultados do consumo médio semanal das dietas, em grama, dos grupos GC e GD, contidos na Figura 2, revelaram para a $2^{\mathrm{a}}$ e $7 \underline{\mathrm{a}}$ semanas do experimento, acréscimo com significância estatística ( $\mathrm{p}<0,05, \mathrm{CV} \%=4,92)$, de consumo da dieta para o grupo de animais que recebeu a ração padrão com o extrato aquoso de amendoim (GD) quando comparado com o grupo alimentado com a dieta normolipídica (GC) e, igualdade estatística para estes grupos na última semana do experimento.

0 Quadro 3 contém os resultados bioquímicos dos animais que receberam dieta normolipídica (grupo controle GC) e dieta normolipídica acrescido com $2 \mathrm{ml} /$ oral/dia de EAA (grupo GD), onde se observa que os animais do grupo GD apresentaram diminuição significativa no nível de colesterol, triglicerídeos e VLDL, quando comparados ao grupo controle $(\mathrm{p}<0,01)$ (Quadro 3). Não se constatou aumento significativo no HDL do grupo GD. Não foi possível calcular os valores de LDL, pois a fórmula de Friedewald não se aplica a valores de triglicerídeos superiores a $399 \mathrm{mg} / \mathrm{dL}$.

Quadro 3. Análises bioquímicas I em ratos que receberam dieta normolipídica (controles) e ratos que além da dieta normolipídica foi acrescentado $2 \mathrm{ml} /$ dia de extrato aquoso de amendoim

\begin{tabular}{lcccc}
\hline \multicolumn{1}{c}{ Parâmetros } & \multicolumn{4}{c}{ Grupos } \\
\cline { 2 - 5 } & $\mathrm{GC}_{\text {normolipídica }}$ & $\mathrm{GD}_{\text {normolipíica+extrato }}$ & & $\mathrm{p}$ \\
\hline Colesterol total (mg/dL) & $90,3 \pm 24,7$ & $62,75 \pm 8,03$ & $46-92$ & $0,009^{* *}$ \\
HDL (mg/dL) & $39,62 \pm 12,67$ & $43,87 \pm 9,52$ & - & $\mathrm{Ns}$ \\
LDL (mg/dL) & - & - & - & \\
Triglicerídeos (mg/dL) & $371,14 \pm 107,2$ & $125,0 \pm 9,33$ & $77-110$ & $<0,009^{* *}$ \\
VLDL (mg/dL) & $75,25 \pm 25,8$ & $24,77 \pm 1.86$ & $15-22$ & $<0,001^{* *}$ \\
AST (U/L) & $131,33 \pm 57,5$ & $104,6 \pm 25,53$ & $39-92$ & $\mathrm{Ns}$ \\
ALT (U/L) & $57,66 \pm 11,04$ & $37,33 \pm 5,14$ & $17-50$ & $0,001^{* *}$ \\
Uréia(mg/dL) & $39,77 \pm 5,56$ & $36,55 \pm 3,57$ & $32-54$ & $\mathrm{Ns}$ \\
Creatinina (mg/dL) & $0,55 \pm 0,05$ & $0,66 \pm 0,09$ & & $0,0237^{*}$ \\
Glicemia(mg/dL) & $116 \pm 13,82$ & $101 \pm 13,22$ & $85-132$ & $0,0365^{*}$
\end{tabular}

*Significativo a $5 \%$ de probabilidade $(\mathrm{p}<0,05)$; ** significativo a $1 \%$ de probabilidade $(\mathrm{p}<0,01) ; \mathrm{ns}=$ não significativo. Valor de Referência ${ }^{1}$.

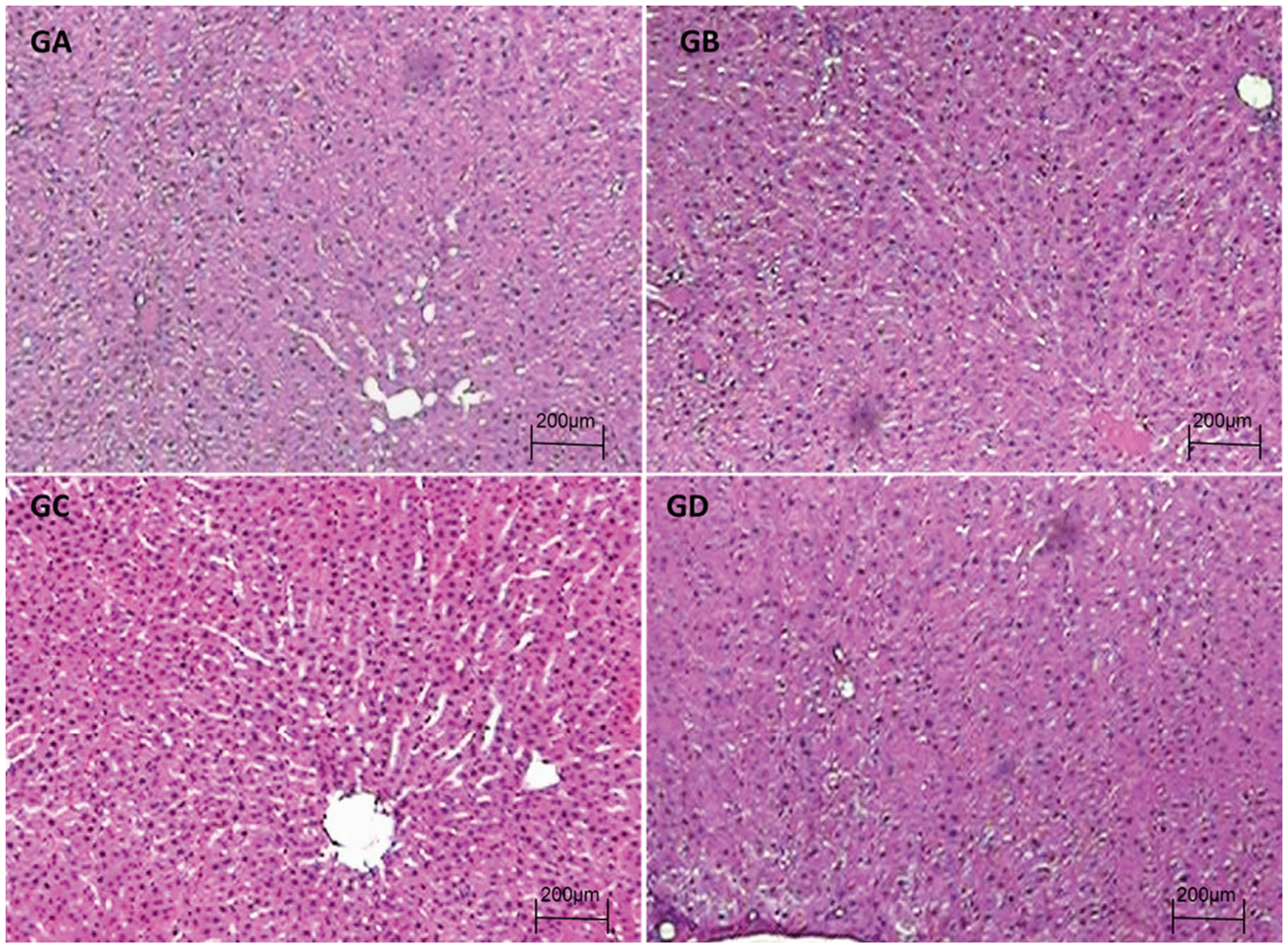

Fig.3. Corte histológico do fígado dos animais do experimento do grupo Hiperlipídica (GA), grupo - Hiperlipídica + EAA (GB), grupo Dieta Normolipídica (GC), grupo Dieta Normolipídica + EAA (GD) em Barra equivale a aproximadamente 200 $\mu \mathrm{m}$. HE, objetiva de 40X. 
Os resultados das dosagens da ureia e AST não apresentaram diferença significativa entre os grupos, entretanto os valores médios de AST ficaram acima dos valores de referência (39-92). Os valores do ALT foram distintos estatisticamente a $1 \%$ de probabilidade; enquanto os de creatinina e glicose a $5 \%(\mathrm{p}<0,05)$, mas por outro lado estes valores encontram-se entre os de referências (85-132mg/dl).

Os fígados dos animais de todos os grupos experimentais não apresentaram diferenças histopatológicas entre si quando analisados à microscopia óptica. 0 parênquima hepático apresentou-se preservado, com os hepatócitos em cordões e seus capilares sinusóides visíveis, com presença de células de Kupfer em aspecto normal, sem aparências patológicas (Fig.3). Histoquimicamente, a técnica de marcação para os lipídios não revelou diferença entre os grupos experimentais, utilizando a coloração "RedOil", o que indica a não deposição patológica de lipídios no fígado. A quantificação da marcação histoquímica em pixels foi confirmada de acordo com a metodologia relatada (Fig.4).

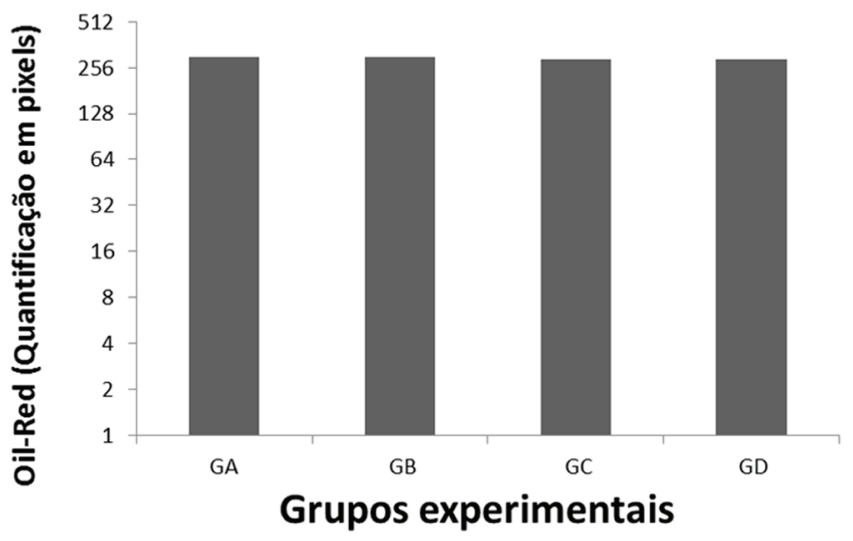

Fig.4. Quantificação em pixels referente a identificação de gordura pela impregnação por Oil red Hiperlipídica (GA), Hiperlipídica + EAA (GB), Dieta Normolipídica (GC), Dieta Normolipídica + EAA (GD).

\section{DISCUSSÃO}

As dislipidemias são fatores de risco para o desenvolvimento de doenças ateroscleróticas - estão entre as principais causas de morbidade em adultos. A aterosclerose é uma doença inflamatória crônica de origem multifatorial, consequente à agressão endotelial, a qual pode ser provocada, por exemplo, pelo aumento de lipoproteínas aterogênicas (LDL, IDL, VLDL, remanescentes de quilomícrons) (Melo et al. 2012). A terapia nutricional é uma medida fundamental na prevenção e no tratamento das dislipidemias, já que o consumo de gordura está relacionado à elevação de colesterol sérico e à maior incidência de aterosclerose aórtica e coronária. A dieta deverá contemplar questões regionais, culturais, socioeconômicas, devendo ser palatável e visualmente atraente, a fim de aumentar a adesão da população (Mattes et al. 2008, Melo et al. 2012).

0 amendoim e seus derivados são ricos em ácidos graxos monoinsaturados, os quais contribuem para diminuir a oxidação, elevar a taxa de HDL e aumentar a captação de LDL pelo fígado. Além de serem ricos em compostos antioxidantes, que reduzem o estresse oxidativo (Basode et al.
2012, Sebei et al. 2013, Naz et al. 2014) e ainda se ligam aos sais biliares e colesterol no tubo digestivo, impedindo sua absorção e prevenindo, portanto, eventos ateroscleróticos (Pereira \& Cardoso 2012). Essas propriedades justificam a redução nos níveis de triglicerídeos nos grupos com intervenção, ou seja, por adição de extrato de amendoim em comparação às dietas normolipídica e hiperlipídica, em que os animais não receberam extrato de amendoim. O EAA promoveu redução de peso e de lipídeos circulantes. Porém sem danificar ou causar diferenças estatísticas a nível hepático. Provavelmente porque o amendoim tenha propriedades de auxiliar na eliminação de gorduras através das fezes, fazendo a gordura seja menos absorvida pelo intestino (McKiernan et al. 2010). Estudos anteriores apresentaram que elevados níveis de triglicerídeos têm emergido como um fator de risco para doença cardiovascular (DCV) independente de colesterol e do LDL (Zhang et al. 2013).

Dietas hipercalóricas ou hiperlipídicas vem sendo utilizadas como modelo de indução de obesidade em ratos Wistar, devido à sua semelhança com a gênese e às respostas metabólicas serem parecidas à obesidade humana, (Silva et al. 2010, Rosini et al. 2012), o que justifica a significativa diferença estatística entre a média do peso corporal inicial e final dos animais do grupo que recebeu dieta hiperlipídica (GA: Peso inicial $=383 \mathrm{~g}$ versus peso final $=424 \mathrm{~g} \mathrm{p}<0,05)$. Resultados similares foram relatados por Khalid \& Siddiqui (2012), os quais ao empregarem em ratos Wistar (submetidos a uma dieta hiperlipídica) o extrato aquoso de uma planta (Punarnava) também rica em flavonóides, polifenóis e antioxidantes, assim como o EAA, obtiverem uma redução nos níveis de gordura corporal (Prenner et al. 2014).

O controle do peso dos ratos dos grupos GB e GD pode estar relacionado além da presença dos antioxidantes, também aos ácidos graxos, os quais promovem a inibição e geração de radicais livres no organismo. Indicando a tendência de melhor controle de peso para os animais suplementados com o extrato, ou seja, esse alimento poderá vir a ser um aliado na perda de peso e doenças cardiovasculares. Alguns pesquisadores relatam que o consumo de amendoim 5 vezes por semana (cerca de 155g / semana) também reduziu o risco de morte por doenças coronárias em 35\% (Sebei et al. 2013, Naz et al. 2014).

As aminotransferases, representadas pela ALT e AST, são enzimas intracelulares de elevada importância para testes hepáticos, avaliando possíveis lesões (Figueira \& Figueiredo 2007). Não foram observadas alterações na AST significantes em nenhum grupo, entretanto houve uma redução da ALT nos dois grupos com intervenção alimentar. Estes resultados indicaram que o EAA tem efeito hepatoprotetor, pois reduziu significativamente $(\mathrm{p}<0,05$ - GB e $\mathrm{p}<0,01$ - GD) os marcadores hepáticos na circulação sistêmica.

Os valores de uréia nos animais com dieta hiperlipídica apresentaram-se menores do que o controle $(\mathrm{p}<0,01)$, entretanto, esse resultado não tem conotação patológica, pois, os valores estão entre os dados de referencia sugerido por Lapchik et al. (2010). Não foi observada diferença significativa ao nível de creatinina na concentração sanguínea dos animais, demonstrando que a ingestão de EAA não alterou patologicamente a função renal. 
Com base nos resultados obtidos, verifica-se, de forma inédita, que o consumo de extrato aquoso de amendoim reduz o perfil lipídico em ratos Wistar em uma dieta hiper- e normolipídica (padrão) sem elevar a glicemia. Mais estudos são necessários para avaliar e dosar os componentes encontrados nesse extrato que possui efeito redutor de gordura.

Agradecimentos.- À Faculdade de Ciências Médicas de Campina Grande pelo apoio durante toda a pesquisa.

\section{REFERÊNCIAS}

Albuquerque E.M.B., Almeida F.A.C., Alves N.M.C. \& Gomes J.P. 2014. Production of "peanut milk" based beverages enriched with umbu and guava pulps. J. Saudi Soc. Agric. Sci. 11(4):61-67.

Almeida F.A.C., Barros Neto J.J.S., Gomes J.P., Alves N.M.C. \& Albuquerque E.M.B. 2014. Leite de amendoim: produto natural, p.110-114. In: Araújo F.D., Baracuhy J.G.V., Francisco P.R.M., Neto Fernandes S. \& Sousa V.A. (Eds), Tecnologias Adaptadas para o Desenvolvimento Sustentável do Semiárido Brasileiro. Vol.1. Epigraf, Campina Grande.

Alper C.M. \& Mattes R.D. 2002. Effects of chronic peanut consumption on energy balance and hedonics. Int. J. Obes. Relat. Metab. Disord. 26(8): 1129-1137.

Araújo A.L. 2007. Correlação entre dieta lipídica polinsaturada e aterogênese. Revta Angiologia Cirúrgica Vascular 5(5):15-22.

Basode R.R., Randolph P., Hurley S. \& Ahmedna M. 2012. Evaluation of hypolipidemic effects of peanut skin-derived polyphenols in rats on Western-diet. Food Chemistry 135(3):1659-1666

Figueira N.A. \& Figueiredo E.A.P. 2007. Icterícias, p.57-67. In: Filgueira N.A., Júnior Costa I.J. \& Leitão S.C.C. (Eds), Condutas em Clínica Médica. $4 \underline{\underline{a}}$ ed. Guanabara Koogan, Rio de Janeiro.

Khalid M. \& Siddiqui H.H. 2012. Evaluation of weight reduction and anti-cholesterol activity of Punarnava root extract against high fat diets induced obesity in experimental rodent. Asian Pac. J. Trop. Biomed. 2(3):1323-1328.

Lapchik V.B.V., Mattaraia V.G.M. \& Ko G.M. 2010. Cuidados e Manejo de Animais de Laboratório. Editora Atheneu, São Paulo.736p.
Machado D.F., Ferreira C.L.L.F., Costa N.M.B. \& Oliveira T.T.O. 2003. Evaluation of the probiotic effect in the modulation of the levels of seric cholesterol and in the weight of the liver of mice fed with rich diet in cholesterol and colic acid. Revta Ciênc. Tecnol. Aliment. 23(2):270-275.

Mattes R.D., Kris-Etherton P.M. \& Foster G.D. 2008. Impact of peanuts and tree nuts on body weight and healthy weight loss in adults. J. Nutrition 138(9):1741S-1745S.

McKiernan F., Lokko P., Kuevi A., Sales R.L., Costa N.M.B., Bressan J., Alfenas R.C.G. \& Mattes R.D. 2010. Effects of peanut processing on body weight and fasting plasma lipids. Brit. J. Nutrition 104(3):418-426.

Melo M.G.D., Doria G.A.A., Serafin M.R. \& Araújo A.A.S. 2012. Reference values of hematological and biochemical rats (Rattus norvegicus Wistar strain) from the central animal house of the Federal University of Sergipe. Scientia Plena 8(9):1-6.

Naz F., Jahan N. \& Sultana N. 2014. Protective effect of peanut (Arachis hypogaea L.) and its combination with propranolol in cardiotoxic rats. J. Bangladesh Soc. Physiol. 9(1):22-30.

Paschoal V., Naves A. \& Fonseca A.B.L. 2007. Nutrição Clínica Funcional dos Princípios à Prática Clínica. 2ª ed. Editora VP, São Paulo 4:212-227.

Pereira R.J. \& Cardoso M.G. 2012. Metabólitos secundários vegetais e benefícios antioxidantes. Minas Gerais. J. Biotec. Biodivers. 3(4):146-152.

Prenner S.B., Mulvey C.K., Ferguson J.F., Rickels M.R., Bhatt A.B. \& Reilly M.P. 2014. Very low density lipoprotein cholesterol associates with coronary artery calcification in type 2 diabetes beyond circulating levels of triglycerides. Atherosclerosis 236(2):244-250.

Rosini T.C., Silva A.S.R. \& Moraes C. 2012. Obesidade induzida por consumo de dieta: modelo em roedores para o estudo dos distúrbios relacionados com a obesidade. Revta Assoc. Méd. Bras. 58(3):383-387.

Sebei K., Gnouma A., Herchi W., Faouzi S. \& Boukhchina S. 2013. Lipids, proteins, phenolic composition, antioxidant and antibacterial activities of seeds of peanuts (Arachis hypogaea L.) cultivated in Tunisia. Biol. Res. 46(3):257-263.

Silva A.S., Pauli J.R., Ropelle E.R., Oliveira A.G., Cintra D.E. \& Souza C.T. 2010. Exercise intensity, inflammatory signaling and insulin resistance in obese rats. Med. Sci. Sports Exerc. 42(12):2180-2188.

Zhang J., Liang R., Wang L., Yan R., Hou R., Gao S. \& Yang B. 2013. Effects of an aqueous extract of Crataegus pinnatifida Bge. var. major N.E.Br. fruit on experimental atherosclerosis in rats. J. Ethnopharmacol. 148(2):563-569. 